\title{
Tracking the epidemiology of antimicrobial drug resistance in hospitals: time to deploy molecular typing
}

The increasing prevalence of antimicrobial drug resistance among hospital-acquired pathogens is a major concern world-wide [1]. Methicillin-resistant strains of Staphylococcus aureus (MRSA) have become endemic and cause outbreaks in many countries. The emergence of MRSA strains with decreased susceptibility to glycopeptides in the USA and Japan [2] raises the issue of lack of effective therapy for infections caused by these pathogens. This is also the case for nosocomial infections with glycopeptide-resistant enterococci [3]. Among gram-negative bacilli, several pathogens - including Klebsiella pneumoniae, Enterobacter aerogenes, Pseudomonas aeruginosa and Acinetobacter baumannii display multi-drug resistance [1]. Resistance to $\beta$-lactam antibiotics, aminoglycosides and fluoroquinolones is increasingly common in these organisms [3, 4]. Hospital-acquired infection caused by antibiotic-resistant organisms is likely to result in prolonged hospital stay and increased mortality. It also contributes to excess hospital costs [1]. Better understanding of the epidemiology of antibiotic resistance is needed urgently. Surveillance and molecular typing are integral to the control of multiresistant pathogens in hospitals.

Widespread use of antimicrobial agents in hospitals and in the community exerts selective pressure on bacteria towards increased resistance. This occurs either through selection of low frequency resistant mutants, acquisition of resistance determinants carried by mobile genetic elements - plasmids and transposons - or reduction of colonisation resistance and replacement of susceptible, endogenous strains by resistant clones. Clonal dissemination of resistant strains is a major determinant of the increasing incidence of nosocomial infection caused by multiresistant strains of $S$. aureus, Enterococcus faecium, $K$. pneumoniae (strains producing extended-spectrum $\beta$-lactamases) $[1,4], E$. aerogenes [5] and Acinetobacter spp. [3]. Hospital outbreaks of multiresistant gram-negative bacteria can result from the interplay of several mechanisms, including the selection of resistance during therapy and subsequent cross-colonisation between patients $[3,5,6]$. Environmental contamination may also play a role as the reservoir, or vehicle of transmission, of multiresistant bacteria. Intensive care departments concentrate a population of critically ill patients who are exposed to invasive procedures and massive use of broad-spectrum antibiotics, thereby creating a breeding ground for the emergence and spread of resistant bacteria [3-5]. Over the past decade, c. $60 \%$ of outbreaks of infection in our tertiary care centre involved antibiotic-resistant organisms and originated in the intensive care units.

Containing the selection and spread of antibioticresistant bacteria in hospitals calls for a multidisciplinary approach, with the implementation of strategies to optimise the use of antimicrobial agents and facilities for the timely detection and control of epidemic strains [7]. The clinical microbiology laboratory plays an important role in this partnership. It should provide accurate and rapid diagnostic methods to identify resistant pathogens in clinical specimens. It should further contribute to the establishment of local surveillance schemes to monitor the prevalence of bacterial resistance to antimicrobial agents and to measure the incidence rates of transmissible multiresistant strains. Targeted surveillance cultures for screening carriers of potentially epidemic organisms - such as MRSA - may be warranted if the institutional policy requires that colonised patients benefit from special isolation precautions or decolonisation therapy. Periodic or continuous analysis of culture results by species and antibiotic resistance profile should provide early warning of clusters of infections or colonisations caused by drug-resistant pathogens [8].

The investigation of potential outbreaks, whenever an increasing prevalence or clustering of resistant organisms is detected, requires the use of discriminating typing systems to understand the dynamics of the resistance problem and to guide infection control interventions [8]. Consider, for instance, a significant increase in the local incidence of infections caused by E. cloacae that are resistant to extended-spectrum cephalosporins. If this is associated with genomically diverse, sporadically occurring clones in patients receiving similar therapeutic regimens, the most likely hypothesis is that selection of resistance (i.e., derepression of chromosomal cephalosporinase) from an endogenous reservoir has occurred. Attention should be focused on reviewing the local antibiotic strategy and patient-related risk factors. In contrast, demonstra- 
tion of transmission of one or several epidemic clones of resistant $E$. cloacae would call for an investigation of the mode of spread and implementation of appropriate control measures. The follow-up of these measures would then include further typing of subsequent isolates to verify the eradication or reduced transmission of the epidemic clones. Unless multiclonal emergence of resistance is differentiated from clonal spread, an effective response to such problems cannot be proposed.

Various high-resolution typing methods are available to analyse a broad range of nosocomial bacterial pathogens [8]. These include: genomic macrorestriction analysis resolved by pulsed-field gel electrophoresis; PCR fingerprinting with arbitrary sequence primers (arbitrarily primed PCR), or primers complementary to multi-copy chromosomal sequences (repetitive-element PCR); and restriction fragment length polymorphism analysis by use of ribosomal probes (ribotyping). The advantages and limitations of these and other methods have been discussed elsewhere $[8,9]$. The use of these tools remains arcane to many clinical microbiologists, but timely access to information on the clonal characteristics of resistant bacteria is necessary for effective surveillance and early control of nosocomial transmission. Although this may be achieved by referral of selected isolates to a reference laboratory in countries with a good public health laboratory infrastructure, this is likely to create delays and potential conflicts of priorities.

Access to modern typing systems varies from country to country, but is often concentrated in larger, teaching institutions. A survey of strategies for the control of resistance in Belgian hospitals in 1997 found that $>80 \%$ of infection control teams used a policy of detection, isolation and decontamination of patient carriers of multiresistant organisms of epidemic potential. However, only $30 \%$ of respondents reported the use of epidemiological typing data to support outbreak detection and control. This suggests that specific isolation measures are sometimes being used for organisms that are not causing local problems. It would, indeed, be difficult to justify the application of the same isolation policies in all these hospitals. Transmission of antibiotic-resistant organisms depends on the epidemic characteristics of various clones, the patient population, local antibiotic prescription patterns and general infection control practices. Moreover, the c. $50 \%$ of hospitals in which a policy is used without access to typing methods cannot reliably evaluate its efficacy; this should be documented by monitoring local rates of importation and transmission of resistant clones in their patient population.

Antibiotic resistance is a global problem. Dissemination of antibiotic-resistant clones occurs not only between medical centres in neighbouring regions, but also between distant countries or continents. Such problems have been linked to inter-institutional transfer of colonised patients or health care personnel [10]. Co-ordination of infection control policies is required and monitoring of geographical spread of epidemic clones must be implemented. To this end, national and international networks of surveillance of antimicrobial resistance are being established. These systems need to integrate molecular typing of representative resistant isolates of major pathogens by means of standardised methods and nomenclature. This would effectively supplement other well-standardised conventional typing schemes, such as phage typing of $S$. aureus. Currently available information technology should allow timely warning of geographical clustering and the spread of epidemic clones to specific sites in the surveillance system. Support for more extensive application of molecular typing tools is needed to achieve a better understanding of the epidemiology of antibiotic-resistant bacteria both at hospital level and national and international levels. Such investment would translate into more efficient control strategies.

MARC J. STRUELENS Department of Microbiology, Université Libre de Bruxelles - Hôpital Erasme, 808 Route de Lennik, B-1070 Bruxelles, Belgium E-mail: marc.struelens@ulb.ac.be

\section{References}

1. Gold HS, Moellering RC. Antimicrobial-drug resistance. $N$ Engl J Med 1996; 335: 1445-1453.

2. Hiramatsu K, Aritaka N, Hamaki H et al. Dissemination in Japanese hospitals of strains of Staphylococcus aureus heterogeneously resistant to vancomycin. Lancet 1997; 350: $1670-1673$.

3. Towner KJ. Clinical importance and antibiotic resistance of Acinetobacter spp. J Med Microbiol 1997; 46: 721-746.

4. Gori A, Espinasse F, Deplano A, Nonhoff C, Nicolas MH Struelens MJ. Comparison of pulsed-field gel electrophoresis and randomly amplified DNA polymorphism analysis for typing extended-spectrum $\beta$-lactamase producing Klebsiella pneumoniae. J Clin Microbiol 1996; 34: 2448-2453.

5. De Gheldre Y, Maes N, Rost F et al. Molecular epidemiology of an outbreak of multidrug-resistant Enterobacter aerogenes infections and in vivo emergence of imipenem resistance. $J$ Clin Microbiol 1997; 35: 152-160.

6. Cheng K, Smyth RL, Govan JRW et al. Spread of $\beta$-lactam resistant Pseudomonas aeruginosa in a cystic fibrosis clinic. Lancet 1996; 348: 639-642.

7. Goldmann DA, Weinstein RA, Wenzel RP et al. Strategies to prevent and control the emergence and spread of antimicrobialresistant microoganisms in hospitals A challenge to hospital leadership. JAMA 1996; 275: 234-240.

8. Struelens MJ. Laboratory methods in the investigation of outbreaks of hospital-acquired infection. In: Emmerson AM, Ayliffe GAJ (eds) Surveillance of nosocomial infections. London, Baillière's Clin Infect Dis 1996; 3: 267-288.

9. Tenover FC, Arbeit RD, Goering RV. How to select and interpret molecular strain typing methods for epidemiological studies of bacterial infections: a review for healthcare epidemiologists. Molecular Typing Working Group of the Society for Healthcare Epidemiology of America. Infect Control Hosp Epidemiol 1997; 18: 426-439.

10. Witte $W$, Kresken M, Braulke C, Cuny C. Increasing incidence and widespread dissemination of methicillin-resistant Staphylococcus aureus (MRSA) in hospitals in central Europe, with special reference to German hospitals. Clin Microbiol Infect 1997; 3: 414-422. 